\title{
AFFECT OF ANTISENSE MCP-1 IN MSPGN RATS
}

\author{
X. Zhang ${ }^{1}, \mathrm{Z} \mathrm{Yi}^{2}$ \\ ${ }^{1}$ Intensive Care Unit, Hunan Children's Hospital, ${ }^{2}$ Nephrology, The Second Xiangya Hospital, Changsha, \\ China
}

Objective: To further clarify whether the antisense MCP-1 have renal protective role in MsPGN rats.

Method: Rats were induced mesangial proliferative glomerulonephritis by injected Thy-1 antibody through tail vain. The infected mesangial cells were transferred the rat kidney via renal artery injection detected by in suit hybridization. $24 \mathrm{~h}$ urinary protein, serum albumin, cholesterol, blood urea nitrogen and serum creatinine were checked by automatic biochemistry analyzer method. Histopathological examination for light microscopy counted by a semiquantitative score.To assess the glomerular hypercellularity, the number of nuclei was counted in ten glomeruli and expressed as the number in per glomeruli. The MCP-1, TGF- $\beta_{1}$ protein secretion were measured by immunohistochemical in paraffin sections. Using RT-PCR evaluate the level of mRNA formation of MCP-1, CCR2, TGF- $\beta_{1}$ in kidney.

Result: In MsPGN rats the infected mesangial cells were detected by in suit hybridization, and the positive cell were stain in glomerular. In antisense group, the glomerular lesion, total cell and $24 \mathrm{~h}$ urinary protein excretion reduce than that of control group. The level of mRNA formation of MCP-1, CCR2, TGF$\beta_{1}$ decreased. The MCP- 1 positive cell in paraffin sections also decreases. But the TGF- $\beta_{1}$ positive cell in paraffin sections still high compare with control.

Conclusion: Mesangial cell can transfer MCP-1cDNA into kidney.Antisense MCP-1 can reduce glomerular proliferation and damage through dresease mRNA formation of MCP-1, CCR2, TGF- $\beta_{1}$ in MsPGN rats.MCP-1 induce extracellular matrix synthesis part by TGF- $\beta_{1}$. 